PROCEEDINGS OF THE

AMERICAN MATHEMATICAL SOCIETY

Volume 128, Number 6, Pages 1617-1625

S 0002-9939(99)05174-6

Article electronically published on October 18, 1999

\title{
INTEGER-VALUED POLYNOMIALS OVER KRULL-TYPE DOMAINS AND PRÜFER $v$-MULTIPLICATION DOMAINS
}

\author{
FRANCESCA TARTARONE
}

(Communicated by Wolmer V. Vasconcelos)

\begin{abstract}
Let $D$ be a domain with quotient field $K$. The ring of integervalued polynomials over $D$ is $\operatorname{Int}(D):=\{f \in K[X] ; f(D) \subseteq D\}$. We characterize the Krull-type domains $D$ such that $\operatorname{Int}(D)$ is a Prüfer $v$-multiplication domain.
\end{abstract}

\section{INTRODUCTION}

One of the open questions about the ring of integer-valued polynomials, $\operatorname{Int}(D):=$ $\{f \in K[X] ; f(D) \subseteq D\}$, has been the characterization of the domains $D$ such that $\operatorname{Int}(D)$ is a Prüfer domain. For Noetherian domains J.L. Chabert has stated that $\operatorname{Int}(D)$ is a Prüfer domain if and only if $D$ is a Dedekind domain with finite residue fields CC, Theorem VI.1.7]. Recently, A. Loper has completely solved this problem providing a description of all domains $D$ such that $\operatorname{Int}(D)$ is Prüfer $[\mathrm{L}$.

Back to J.L. Chabert's characterization, we can ask whether $\operatorname{Int}(D)$ still satisfies some multiplicative ideal properties when $D$ is a Dedekind domain, not necessarily with all residue fields finite. This was exactly the starting point of this work. We obtain the answer to this question as a corollary of a more general statement.

In multiplicative ideal theory an important role is assumed by the set of the $t$-ideals of a domain. Many definitions of classical domains have been revisited and generalized by requiring that just $t$-ideals satisfy certain properties. For instance, Prüfer v-multiplication domains (PvMD) are domains for which the localization at each $t$-prime ideal is a valuation ring. Whence they generalize Prüfer domains, for which this last property holds for all prime ideals.

It is known that a domain $D$ is a $\mathrm{P} v \mathrm{MD}$ if and only if $D[X]$ is a $\mathrm{P} v \mathrm{MD}$. In this work we ask when $\operatorname{Int}(D)$ is a $\mathrm{P} v \mathrm{MD}$.

First, we characterize the valuation domains $V$ such that $\operatorname{Int}(V)$ is a $\mathrm{P} v \mathrm{MD}$ (Theorem 1.1). We also give a necessary condition for any domain $D$, showing that if $\operatorname{Int}(D)$ is a $\mathrm{P} v \mathrm{MD}$, then $D$ is a $\mathrm{P} v \mathrm{MD}$ (Proposition 3.1). From the analysis carried on $\operatorname{Int}(V)$, when $V$ is a valuation domain, it easily follows that the condition of Proposition 3.1 is not, in general, sufficient.

Received by the editors March 5, 1998 and, in revised form, July 22, 1998.

1991 Mathematics Subject Classification. Primary 13A15, 13A18, 13B25; Secondary 13B30.

The author would like to thank Prof. Stefania Gabelli who introduced her to this topic and who gave her useful advice for this work. She also would like to thank the Laboratoire des Mathématiques de la Faculté des Sciences de Saint-Jérôme in Marseille where she is attending a Post-Doc research program and Prof. P.-J. Cahen who carefully read this paper providing valuable suggestions. 
We consider Krull-type domains, that is, domains $D$ which can be realized as an intersection of the type

$$
D=\bigcap_{p \in \mathcal{P}} D_{p}
$$

where $\mathcal{P} \subseteq \operatorname{Spec}(D), D_{p}$ is a valuation domain and the intersection is locally finite (that is, each nonzero element $x \in D$ belongs to finitely many $p \in \mathcal{P}$ ). For example, Krull domains or generalized Krull domains are domains of Krull-type [Gi1, p. 524]. We characterize the Krull-type domains $D$ such that $\operatorname{Int}(D)$ is a $\mathrm{P} v \mathrm{MD}$ (Theorem $3.2)$.

As a corollary we describe the Noetherian domains $D$ such that $\operatorname{Int}(D)$ is a $\mathrm{P} v \mathrm{MD}$. Precisely, we prove that, if $D$ is Noetherian, $\operatorname{Int}(D)$ is a $\mathrm{P} v \mathrm{MD}$ if and only if $D$ is a Krull domain, that is, $D$ is integrally closed (Corollary 3.3).

When we restrict to one-dimensional Noetherian domains, we show that $\operatorname{Int}(D)$ is a $\mathrm{P} v \mathrm{MD}$ if and only if $D$ is Dedekind (Corollary 3.4), giving evidence to the fact that $\operatorname{Int}(D)$ verifies interesting multiplicative properties for any Dedekind domain $D$, also when the residue fields are not all finite.

\section{When is $\operatorname{Int}(V)$ a Prüfer $v$-multiplication domain?}

In this first section we give a characterization of the valuation domains $V$ such that $\operatorname{Int}(V)$ is a $\mathrm{P} v \mathrm{MD}$. We briefly recall some definitions and general properties about $v$-ideals and $t$-ideals. We refer to [Gi1, § 32] or [J] for the general theory about the $t$-operation and the $v$-operation.

Let $D$ be a domain and $\mathfrak{F}(D)$ the set of its fractional ideals. Let $I \in \mathfrak{F}(D)$.

The $v$-closure of $I$ is the ideal $I_{v}:=\left(I^{-1}\right)^{-1}=(D:(D: I))$, and $I$ is $v$-finite if there exists a finitely generated ideal $J \in \mathfrak{F}(D)$ such that $I_{v}=J_{v}$.

The $t$-closure of $I$ is $I_{t}:=\bigcup J_{v}$ where $J$ ranges over the set of the finitely generated ideals contained in $I$.

The ideal $I$ is a $t$-ideal if $I=I_{t}$ and $I$ is $t$-invertible if there exists $J \in \mathfrak{F}(D)$ such that $(I J)_{t}=D$.

If $I$ is an integral ideal, then $I$ is a $t$-prime if it is a prime and a $t$-ideal.

A $t$-maximal ideal is a maximal element among the integral $t$-ideals of $D$. A $t$-maximal ideal is prime and each integral $t$-ideal is contained in a $t$-maximal ideal. We denote by $\Theta$ the set of $t$-maximal ideals of $D$.

The $t$-dimension of $D(t-\operatorname{dim}(D))$ is defined as the supremum of the lengths of chains of $t$-primes. If this supremum is not bounded, then the $t$-dimension of $D$ is infinite.

In a Prüfer domain each ideal is a $t$-ideal. A domain $D$ is a Prüfer v-multiplication domain $(\mathrm{P} v \mathrm{MD})$ if the following equivalent conditions hold:

(i) a fractional ideal of $D$ is $t$-invertible if and only if it is $v$-finite;

(ii) $D_{p}$ is a valuation domain for each $t$-prime ideal $p$ of $D$.

(iii) $D_{m}$ is a valuation domain for each $t$-maximal ideal $m$ of $D$.

In particular, Prüfer domains are Prüfer $v$-multiplication.

For a more detailed account about PvMD's we refer the reader to [K1] and [K2].

Theorem 1.1. Let $V$ be a valuation domain with maximal ideal $m$. Then, $\operatorname{Int}(V)$ is a PvMD if and only if $\operatorname{Int}(V)=V[X]$ (that is, $V$ has infinite residue field or nonprincipal maximal ideal [CC, Proposition I.3.16]) or $V$ is a DVR with finite residue field. 
Proof. If $\operatorname{Int}(V)=V[X]$, then $\operatorname{Int}(V)$ is a $\mathrm{P} v \mathrm{MD}$ by [K1, Theorem 3.7] and since $V$ is a $\mathrm{P} v \mathrm{MD}$.

We suppose that $\operatorname{Int}(V) \neq V[X]$. Thus $m:=\pi V$ is principal and has finite residue field [CC, Proposition I.3.16].

If $V$ is one-dimensional, then $V$ is a DVR with finite residue field and $\operatorname{Int}(V)$ is a Prüfer domain $\mathrm{CC}$ Theorem VI.1.7]. Hence $\operatorname{Int}(V)$ is a $\mathrm{P} v \mathrm{MD}$.

We suppose that $\operatorname{dim}(V)>1$ and show that $\operatorname{Int}(V)$ is not a $\mathrm{P} v \mathrm{MD}$. From [CH] we have that all the prime ideals of $\operatorname{Int}(V)$ above $m$ are maximal and so they are minimal over $m \operatorname{Int}(V)$. But, $m \operatorname{Int}(V)=\pi \operatorname{Int}(V)$ is a $t$-ideal (because it is principal) and its minimal primes are also $t$-ideals [J, Corollaire 3, p. 31]. Whence all the prime ideals of $\operatorname{Int}(V)$ above $m$ are $t$-ideals. The ideal $\mathfrak{M}_{0}:=\{f \in \operatorname{Int}(V) ; f(0) \in m\}$ of $\operatorname{Int}(V)$ is prime and it is a $t$-ideal (since it is above $m$ ). There exists, at least, one prime ideal $q$ in $V$ such that $(0) \subsetneq q \subsetneq m$. The ideals $q[X]=q V_{q}[X]=$ $q V_{q}[X] \cap \operatorname{Int}(V)$ and $\beta_{X}:=X K[X] \cap \operatorname{Int}(V)=\{f \in \operatorname{Int}(V) ; f(0)=0\}$ are primes and they are both contained in $\mathfrak{M}_{0}$. Thus they lift to $\operatorname{Int}(V)_{\mathfrak{M}_{0}}$ and they are incomparable. In fact, $\beta_{X} \nsubseteq \nsubseteq q[X]$ since $X \in \beta_{X}$ whence $X \notin q[X]$, and $q[X] \nsubseteq \beta_{X}$ since $q[X]$ contains nonzero constants. This implies that $\operatorname{Int}(V)_{\mathfrak{M}_{0}}$ is not a valuation domain (since its prime spectrum is not linearly ordered). Therefore $\operatorname{Int}(V)$ is not a $\mathrm{P} v \mathrm{MD}$ (by definition).

\section{Some Results about $t$-IDEals of $\operatorname{Int}(D)$}

In this section we give some results which relate the $t$-closure of the ideals of $\operatorname{Int}(D)$ to the $t$-closure of their contraction in $D$.

Proposition 2.1. Let $D$ be a domain. If $I$ is an ideal of $D$, then $(\operatorname{Int}(D))_{t} \cap D=$ $I_{t}$. In particular, if $\mathfrak{A}$ is a t-ideal of $\operatorname{Int}(D)$, then $\mathfrak{A} \cap D$ is a $t$-ideal of $D$.

Proof. By definition, $(I \operatorname{Int}(D))_{t}:=\bigcup J_{v}$, where $J$ ranges over the finitely generated ideals of $\operatorname{Int}(D)$ contained in $I \operatorname{Int}(D)$. Since each of these $J$ is included in a finitely generated ideal $\left(a_{1}, \ldots, a_{r}\right) \operatorname{Int}(D)$, for $a_{1}, \ldots, a_{r} \in I$, and $J_{v} \subseteq$ $\left(\left(a_{1}, \ldots, a_{r}\right) \operatorname{Int}(D)\right)_{v}$, the union above can be restricted to ideals of $\operatorname{Int}(\mathrm{D})$ generated by finitely many elements $a_{1}, \ldots, a_{r}$ of $I$. Notice that

$$
\begin{gathered}
\left(\left(a_{1}, \ldots, a_{r}\right) \operatorname{Int}(D)\right)^{-1}=\left(\operatorname{Int}(D):\left(a_{1}, \ldots, a_{r}\right) \operatorname{Int}(D)\right) \\
=\left(1 / a_{1}\right) \operatorname{Int}(D) \cap \ldots \cap\left(1 / a_{r}\right) \operatorname{Int}(D)
\end{gathered}
$$

and

$$
\left(\left(a_{1}, \ldots, a_{r}\right) D\right)^{-1}=\left(D:\left(a_{1}, \ldots, a_{r}\right) D\right)=\left(1 / a_{1}\right) D \cap \ldots \cap\left(1 / a_{r}\right) D .
$$

We will show that, if $a_{1}, \ldots, a_{r} \in D$, then

$$
\left(\left(a_{1}, \ldots, a_{r}\right) \operatorname{Int}(D)\right)_{v} \cap D=\left(\left(a_{1}, \ldots, a_{r}\right) D\right)_{v} .
$$

The inclusion " $\subseteq$ " is easy to check. In fact, if $x \in\left(\left(a_{1}, \ldots, a_{r}\right) \operatorname{Int}(D)\right)_{v} \cap D$, then $x\left(\left(1 / a_{1}\right) \operatorname{Int}(D) \cap \ldots \cap\left(1 / a_{r}\right) \operatorname{Int}(D)\right) \subseteq \operatorname{Int}(D)$, so that $x\left(\left(1 / a_{1}\right) D \cap \ldots \cap\left(1 / a_{r}\right) D\right) \subseteq$ $D$, that is, $x \in\left(\left(a_{1}, \ldots, a_{r}\right) D\right)_{v}$.

The opposite inclusion " $\supseteq$ " holds for the following argument.

If $x \in\left(\left(a_{1}, \ldots, a_{r}\right) D\right)_{v}$, then $x\left(\left(1 / a_{1}\right) D \cap \ldots \cap\left(1 / a_{r}\right) D\right) \subseteq D$. Moreover, if $f \in\left(1 / a_{1}\right) \operatorname{Int}(D) \cap \ldots \cap\left(1 / a_{r}\right) \operatorname{Int}(D)$, then $f(d) \in\left(1 / a_{1}\right) D \cap \ldots \cap\left(1 / a_{r}\right) D$, for each $d \in D$. Thus $x f(d) \in x\left(\left(1 / a_{1}\right) D \cap \ldots \cap\left(1 / a_{r}\right) D\right) \subseteq D$ and $x f \in \operatorname{Int}(D)$, that is, $x \in\left(\left(a_{1}, \ldots, a_{r}\right) \operatorname{Int}(D)\right)_{v} \cap D$. 
We have seen that $(I \operatorname{Int}(D))_{t}$ is the union of the ideals $\left(\left(a_{1}, \ldots, a_{r}\right) \operatorname{Int}(D)\right)_{v}$, where $a_{1}, \ldots, a_{r} \in I$. Therefore $(I \operatorname{Int}(D))_{t} \cap D=I_{t}$, since $\left(\left(a_{1}, \ldots, a_{r}\right) \operatorname{Int}(D)\right)_{v} \cap$ $D=\left(\left(a_{1}, \ldots, a_{r}\right) D\right)_{v}$ and $I_{t}$ is the union of the ideals $\left(\left(a_{1}, \ldots, a_{r}\right) D\right)_{v}$, for $a_{1}, \ldots, a_{r}$ $\in I$.

If $\mathfrak{A}$ is a $t$-ideal of $\operatorname{Int}(D)$, then $((\mathfrak{A} \cap D) \operatorname{Int}(D))_{t}=(\mathfrak{A} \cap D)_{t}$. But, $(\mathfrak{A} \cap D) \operatorname{Int}(D) \subseteq$ $\mathfrak{A}$ and, since $\mathfrak{A}$ is a $t$-ideal $((\mathfrak{A} \cap D) \operatorname{Int}(D))_{t} \subseteq \mathfrak{A}$. By intersection with $D$, it follows that $\mathfrak{A} \cap D \subseteq((\mathfrak{A} \cap D) \operatorname{Int}(D))_{t} \cap D \subseteq \mathfrak{A} \cap D$, that is, $\mathfrak{A} \cap D$ is a $t$-ideal.

Corollary 2.2. Let $D$ be a domain. Then

(a) if $\mathfrak{Q}$ is a t-prime ideal of $\operatorname{Int}(D)$, then $q:=\mathfrak{Q} \cap D$ is a t-prime ideal of $D$;

(b) each t-maximal ideal of $D$ is the contraction of a t-maximal ideal of $\operatorname{Int}(D)$.

Proof. (a) directly follows from the fact that, if $\mathfrak{Q}$ is a prime ideal of $\operatorname{Int}(D)$, then $q:=\mathfrak{Q} \cap D$ is a prime ideal of $D$ and that, by Proposition 2.1, if $\mathfrak{Q}$ is a $t$-ideal, then $q$ is a $t$-ideal too.

(b) We have that if $p$ is a $t$-prime ideal of $D$, then $p \operatorname{Int}(D)$ is contained in a $t$-prime ideal of $\operatorname{Int}(D)$. In fact, if it was $(p \operatorname{Int}(D))_{t}=\operatorname{Int}(D)$, by Proposition 2.1 we would have that $p_{t}=(p \operatorname{Int}(D))_{t} \cap D=\operatorname{Int}(D) \cap D=D$, against the assumption that $p$ is a $t$-ideal. Hence $p \operatorname{Int}(D)$ is contained in a $t$-maximal ideal $\mathfrak{P}$ of $\operatorname{Int}(D)$. From (a), $\mathfrak{P} \cap D$ is a $t$-prime ideal of $D$ and, for the maximality of $p, \mathfrak{P} \cap D=p$.

In [CC, Propositon I.2.8] it is proved that if $D$ is a Krull domain and $p$ is a height-one prime ideal of $D$, then $\operatorname{Int}(D)_{p}=\operatorname{Int}\left(D_{p}\right)$. We show that if $D$ is a domain representable as a locally finite intersection of overrings $D_{p}$, where $p$ belongs to a subset $\mathcal{P} \subseteq \operatorname{Spec}(D)$, then the equality above holds for each $p \in \mathcal{P}$.

Proposition 2.3. Let $D$ be a domain and let us suppose that $D$ has a locally finite representation, $D=\bigcap_{p \in \mathcal{P}} D_{p}$, where $\mathcal{P} \subseteq \operatorname{Spec}(D)$. Then, $\operatorname{Int}(D)_{p}=\operatorname{Int}\left(D_{p}\right)$, for each $p \in \mathcal{P}$.

Proof. We fix $q \in \mathcal{P}$. We always have the containment $\operatorname{Int}(D)_{q} \subseteq \operatorname{Int}\left(D_{q}\right)$ CC Proposition I.2.2].

Now, let $f \in \operatorname{Int}\left(D_{q}\right)$ and let us see that there exists $s \in D, s \notin q$, such that $s f(D) \subseteq D_{p}$ for all $p \in \mathcal{P}$. In this case, we would have $s f(D) \subseteq \bigcap_{p \in \mathcal{P}} D_{p}=D$, and we would finally conclude that $f \in \operatorname{Int}(D)_{q}$.

From the locally finite character of the intersection $\bigcap_{p \in \mathcal{P}} D_{p}$, we have that $f \in$ $D_{p}[X]$ for all $p \in \mathcal{P}$ but finitely many, namely $p_{1}, \cdots, p_{n}$, so that $f(D) \subseteq D_{p}$ for all $p \in \mathcal{P}$ but $p \neq p_{1}, \cdots, p_{n}$. Since $f \in \operatorname{Int}\left(D_{q}\right)$, then $f(D) \subseteq\left(D_{q}\right)_{p_{i}}$, for $i=1, \cdots, n$.

If $p_{i} \subseteq q$, then $\left(D_{q}\right)_{p_{i}}=D_{p_{i}}$, whence $f(D) \subseteq D_{p_{i}}$.

If $p_{i} \not \subset q$, we consider the family $\mathcal{P}^{\prime}$ of all the prime ideals $p^{\prime}$ of $D$ such that $p^{\prime} \subset p_{i} \cap q$. Then, $\left(D_{q}\right)_{p_{i}}=S_{i}^{-1} D=\bigcap_{p^{\prime} \in \mathcal{P}^{\prime}} D_{p^{\prime}}$. For $p^{\prime} \in \mathcal{P}^{\prime}, p^{\prime}$ is distinct from $q$, whence $p^{\prime}$ is not maximal and $\operatorname{Int}\left(D_{p^{\prime}}\right)=D_{p^{\prime}}[X]$. Therefore, $f(D) \subseteq D_{q} \subseteq D_{p^{\prime}}$. Hence $f \in \bigcap_{p^{\prime} \in \mathcal{P}^{\prime}} \operatorname{Int}\left(D_{p^{\prime}}\right)=\bigcap_{p^{\prime} \in \mathcal{P}^{\prime}} D_{p^{\prime}}[X]=S_{i}^{-1} D[X]$. There exists $s_{i} \in D$, $s_{i} \notin q$, such that $s_{i} f \in D_{p_{i}}[X]$, that is, $s_{i} f(D) \subseteq D_{p_{i}}$. If we take the product $s:=s_{1} \cdots s_{n}$, then $s \in D, s \notin q$, and $s f(D) \subseteq D$.

M. Griffin proved that a domain $D$ can always be represented as follows:

$$
D=\bigcap_{p \in \Theta} D_{p} \quad[\mathrm{Gr}, \text { Proposition 4]. }
$$

Moreover, from [Ch, Corollaire 1], we have that $\operatorname{Int}(D)=\bigcap_{p \in \Theta} \operatorname{Int}\left(D_{p}\right)$. 
Inside $\Theta$ we can consider the subset $\Theta_{0}$ of the ideals $p$ such that $\operatorname{Int}(D)_{p} \neq D_{p}[X]$ and the subset $\Theta_{1}$ of the ideals $p$ such that $\operatorname{Int}(D)_{p}=D_{p}[X]$. In this last case, we observe that the prime ideals of $\operatorname{Int}(D)$ above $p$ are contractions of the prime ideals of $D_{p}[X]$ above $p D_{p}$. They are of the type $J_{p}:=p D_{p}[X] \cap \operatorname{Int}(D)$ or they strictly contain $J_{p}$ and they are upper to $p$.

If $D$ is a $\mathrm{P} v \mathrm{MD}$ (that is, $D_{p}$ is a valuation domain for each $p \in \Theta$ ) and, further, the representation (2.I) is locally finite, then $D$ is a Krull-type domain (that is, $D$ is a locally finite intersection of valuation overrings $D_{p}$, where $p \in \operatorname{Spec}(D)$ ). More precisely, in [Gr. Theorem 7] the author proves that Krull-type domains are exactly $\mathrm{P} v \mathrm{MD}$ 's for which the representation (2.I) is locally finite (that is, $\mathrm{P} v \mathrm{MD}$ 's such that each nonzero element $x$ belongs to finitely many $t$-maximal ideals).

Proposition 2.4. Let $D$ be a Krull-type domain and let us suppose that $p$ has height one for each $p \in \Theta_{0}$. If $p$ is a prime ideal of $D$ such that $\operatorname{Int}(D)_{p}=D_{p}[X]$, then the prime ideals of $\operatorname{Int}(D)$ which are upper to $p$ are not $t$-ideals.

Proof. If $p$ is not a $t$-prime ideal of $D$, from Corollary 2.2 (a) no prime ideal of $\operatorname{Int}(D)$ above $p$ is a $t$-ideal.

Let us suppose that $p$ is a $t$-prime ideal of $D$ and let $P$ be a prime ideal of $\operatorname{Int}(D)$ upper to $p$. Thus $P \cap D[X]$ is a prime ideal of $D[X]$ upper to $p$ and $p[X] \subsetneq P \cap D[X]$. We claim that $(P \cap D[X])_{t}=D[X]$. Since $D$ is integrally closed, by [HZ, Lemma 4.5] $P$ is not a $t$-ideal. We want to see that $P$ is not included in any other $t$-prime ideal of $D[X]$ (which would imply that $(P \cap D[X])_{t}=D[X]$ ).

If $\mathfrak{P}$ is a $t$-prime ideal of $D[X]$ containing $P$, then $\mathfrak{P}=q[X]$, for some $t$-prime ideal $q$ of $D$. Thus $P D_{q}[X] \subset q D_{q}[X]$. But $D_{q}$ is a valuation domain (because $D$ is, in particular, a $\mathrm{P} v \mathrm{MD}$ ), and the extended prime ideals of $D_{q}[X]$ can only contain extended primes Gi1, Theorem 19.5], so that we cannot have the inclusion $P D_{q}[X] \subset q D_{q}[X]$. Therefore, there exists a finitely generated ideal $I \subseteq P \cap D[X]$ such that $I_{t}=I_{v}=D[X]$. By the fact that $D=\bigcap_{p \in \Theta} D_{p}$ is a locally finite intersection, we can choose $I$ such that $I \cap D \not \subset q$ for each $t$-prime ideal $q$ of $D$ that does not contain $p$. We set $J:=I \operatorname{Int}(D)$. We have that $J_{q}=\operatorname{Int}(D)_{q}=\operatorname{Int}\left(D_{q}\right)$, for each $q \in \Theta$ and $q \nsupseteq p$.

Moreover we have that:

$$
\begin{gathered}
J_{v}=(\operatorname{Int}(D):(\operatorname{Int}(D): J))=\left(\bigcap_{q \in \Theta} \operatorname{Int}(D)_{q}: \bigcap_{q \in \Theta}(\operatorname{Int}(D): J)_{q}\right) \\
=\left(\bigcap_{q \in \Theta} \operatorname{Int}\left(D_{q}\right): \bigcap_{q \in \Theta}\left(\operatorname{Int}\left(D_{q}\right): J_{q}\right)\right) \supseteq \bigcap_{q \in \Theta}\left(\operatorname{Int}\left(D_{q}\right):\left(\operatorname{Int}\left(D_{q}\right): J_{q}\right)\right),
\end{gathered}
$$

where $(\operatorname{Int}(D): J)_{q}=\left(\operatorname{Int}(D)_{q}: J_{q}\right)$ since $J$ is finitely generated and $\operatorname{Int}(D)_{q}=$ $\operatorname{Int}\left(D_{q}\right)$ from Proposition 2.3.

If $q \nsupseteq p$, then

$$
\left(\operatorname{Int}\left(D_{q}\right):\left(\operatorname{Int}\left(D_{q}\right): J_{q}\right)\right)=\left(\operatorname{Int}\left(D_{q}\right):\left(\operatorname{Int}\left(D_{q}\right): \operatorname{Int}\left(D_{q}\right)\right)\right)=\operatorname{Int}\left(D_{q}\right) .
$$

If $q \supseteq p$, then $\operatorname{Int}\left(D_{q}\right)=D_{q}[X]$ (because either $q=p$ or $q$ does not have height-one; hence $\left.q \in \Theta_{1}\right)$ and $J_{q}=I_{q}$. Thus we have:

$$
\begin{gathered}
\left(\operatorname{Int}\left(D_{q}\right):\left(\operatorname{Int}\left(D_{q}\right): J_{q}\right)\right)=\left(D_{q}[X]:\left(D_{q}[X]: J_{q}\right)\right) \\
=\left(D_{q}[X]:\left(D_{q}[X]: I_{q}\right)\right) \supseteq(D[X]:(D[X]: I))_{q}=\left(I_{v}\right)_{q}=D_{q}[X]=\operatorname{Int}\left(D_{q}\right) .
\end{gathered}
$$


Therefore $J_{v} \supseteq \bigcap_{q \in \Theta} \operatorname{Int}\left(D_{q}\right)=\operatorname{Int}(D)$. Hence $J_{v}=\operatorname{Int}(D)$ and $P$ is not a $t$-prime ideal.

As a corollary of these results we describe all the $t$-prime ideals of $\operatorname{Int}(D)$ when $D$ is a Krull-type domain satisfying the hypotheses of Proposition 2.4. We briefly recall some results about the prime spectrum of $\operatorname{Int}(D)$.

- If $D$ is a domain, all the prime ideals of $\operatorname{Int}(D)$ upper to (0) are of the type $\beta_{q}:=q K[X] \cap \operatorname{Int}(D)$, where $q$ is an irreducible polynomial of $K[X]$ CC, Corollary V.1.2].

- If $D$ is a domain, $p$ is a prime ideal of $D$ such that $D_{p}$ is a DVR with finite residue field and such that $\operatorname{Int}(D)_{p}=\operatorname{Int}\left(D_{p}\right)$, then all the prime ideals of $\operatorname{Int}(D)$ above $p$ are of the type $p_{\alpha}:=\left\{f \in \operatorname{Int}(D) ; f(\alpha) \in \widehat{p D_{p}}\right\}$, where $\widehat{D_{p}}$ is the $p$-adic completion of $D_{p}$ and $\alpha \in \widehat{D_{p}}[\mathrm{CC}, \mathrm{Ch} . \mathrm{V}]$.

Corollary 2.5. With the notation above, let $D$ be a Krull-type domain and let us suppose that $p$ has height one, for each $p \in \Theta_{0}$. Then, the t-prime ideals of $\operatorname{Int}(D)$ are the following:

- the primes upper to (0), that is, the ideals of the type $\beta_{q}:=q K[X] \cap \operatorname{Int}(D)$, where $q$ is an irreducible polynomial of $K[X]$;

- the primes of the type $J_{p}:=p D_{p}[X] \cap \operatorname{Int}(D)$, where $p$ is a t-prime ideal of $D$ and $\operatorname{Int}(D)_{p}=D_{p}[X]$ (that is, $p \in \Theta_{1}$ );

- the primes above some prime ideal $p \in \Theta_{0}$, that is, the ideals of the type $p_{\alpha}$, with $\alpha \in \widehat{D_{p}}$.

Proof. From Corollary 2.2 (a) we only have to consider the prime ideals of $\operatorname{Int}(D)$ which are above some $t$-prime ideal of $D$.

By [J], Corollaire 3, p. 31] the primes upper to (0) are $t$-ideals (since they are minimal over the principal ideal (0)).

If $p$ is a $t$-prime ideal of $D$ such that $\operatorname{Int}(D)_{p}=D_{p}[X]$, by Proposition 2.4 the prime ideals of $\operatorname{Int}(D)$ which are upper to $p$ are not $t$-ideals.

The prime ideals of the type $J_{p}:=p D_{p}[X] \cap \operatorname{Int}(D)$ are $t$-ideals. In fact, $p D_{p}$ is a $t$-ideal of $D_{p}$, since $D_{p}$ is a Prüfer domain; then $p D_{p}[X]$ is a $t$-ideal in $D_{p}[X]$, by [K1, Corollary 2.3], and $J_{p}$ is a $t$-ideal of $\operatorname{Int}(D)$ because it is the contraction of a $t$-ideal from a localization overring [K1, Lemma 3.17].

Finally, if $p \in \Theta_{0}$, applying Proposition 2.3 to the locally finite intersection $D=\bigcap_{p \in \Theta} D_{p}$, we have that $\operatorname{Int}(D)_{p}=\operatorname{Int}\left(D_{p}\right)$. Moreover, $D_{p}$ is one-dimensional and, since $\operatorname{Int}(D)_{p} \neq D_{p}[X], D_{p}$ is a DVR with finite residue field [CC, Proposition I.3.16]. Thus all the prime ideals of $\operatorname{Int}(D)$ above $p$ are of the type $p_{\alpha}$, with $\alpha \in \widehat{D_{p}}$ and they are contractions of prime ideals of $\operatorname{Int}\left(D_{p}\right)$. Since $\operatorname{Int}\left(D_{p}\right)$ is a Prüfer domain [CC, Theorem VI.1.7], each ideal in $\operatorname{Int}\left(D_{p}\right)$ is a $t$-ideal and its contraction to $\operatorname{Int}(D)$ is still a $t$-ideal [K1, Lemma 3.17].

\section{Krull-type domains $D$ Such that $\operatorname{Int}(D)$ is a $\mathrm{P} v \mathrm{MD}$}

We start this section giving a necessary condition on a domain $D$ for $\operatorname{Int}(D)$ to be a $\mathrm{P} v \mathrm{MD}$.

Proposition 3.1. Let $D$ be a domain and let us suppose that $\operatorname{Int}(D)$ is a PvMD. Then, $D$ is a PvMD.

Proof. If $p$ is a $t$-prime ideal of $D$, then $p$ is contained in a $t$-maximal ideal $p^{\prime}$. From Corollary $2.2(\mathrm{~b})$, there exists a $t$-maximal ideal $\mathfrak{P}$ of $\operatorname{Int}(D)$ such that $\mathfrak{P} \cap D=p^{\prime}$. 
Thus $p \operatorname{Int}(D) \subseteq p^{\prime} \operatorname{Int}(D) \subseteq \mathfrak{P}$. Since $\operatorname{Int}(D)$ is a $\mathrm{P} v \mathrm{MD}$, $\operatorname{Int}(D)_{\mathfrak{P}}$ is a valuation domain. Moreover, $\operatorname{Int}(D)_{\mathfrak{P}} \cap K=D_{\mathfrak{P} \cap D}=D_{p^{\prime}}$ is also a valuation domain, so that $D_{p}$ is a valuation domain, because $D_{p^{\prime}} \subseteq D_{p}$. Therefore $D$ is a $\mathrm{P} v \mathrm{MD}$ by definition.

The condition that we have given in Proposition 3.1 is not, in general, sufficient to have that $\operatorname{Int}(D)$ is a $\mathrm{P} v \mathrm{MD}$. In fact, for example, in Theorem 1.1 we have seen that there exist valuation domains (which are Prüfer domains, and so P $v$ MDs) such that $\operatorname{Int}(V)$ is not a $\mathrm{P} v \mathrm{MD}$.

We observe that if $D$ is a domain, $p$ is a prime ideal of $D$ and $D_{p}$ is a valuation domain, then $p$ is a $t$-prime ideal. In fact, $p D_{p}$ is a $t$-ideal (since $D_{p}$ is a Prüfer domain), and $p=p D_{p} \cap D$. By [K1, Lemma 3.17] $p$ is a $t$-prime ideal. It directly follows that, if $D$ is a $\mathrm{P} v \mathrm{MD}$ and $q$ is a prime ideal contained in a $t$-prime $p$, then $q$ is a $t$-prime too. In fact, we have that $D_{q} \supseteq D_{p}$ and $D_{p}$ is a valuation domain. Therefore $D_{q}$ is also a valuation domain and $q$ is a $t$-prime ideal. Thus $t-\operatorname{dim}(D)=$ $\sup \left\{\operatorname{dim}\left(D_{p}\right) ; p \in \Theta\right\}$.

In the next theorem we characterize the Krull-type domains such that $\operatorname{Int}(D)$ is a $\mathrm{P} v \mathrm{MD}$.

Theorem 3.2. Let $D$ be a Krull-type domain. With the notation above, $\operatorname{Int}(D)$ is a PvMD if and only if $D_{p}$ is one dimensional for each $p \in \Theta_{0}$. Moreover,

$$
t-\operatorname{dim}(\operatorname{Int}(D))=\sup \{2, t-\operatorname{dim}(D)\}=\sup \left\{2, \sup _{p \in \Theta}\left\{\operatorname{dim}\left(D_{p}\right)\right\}\right\} .
$$

Proof. Let us suppose that $\operatorname{Int}(D)$ is a $\mathrm{P} v \mathrm{MD}$ and that there exists a prime ideal $p \in \Theta_{0}$ such that $D_{p}$ has dimension $n$, with $n>1$. Since $\operatorname{Int}(D)_{p} \neq D_{p}[X]$, then also $\operatorname{Int}\left(D_{p}\right) \neq D_{p}[X]$ (because, in general, $D_{p}[X] \subseteq \operatorname{Int}(D)_{p} \subseteq \operatorname{Int}\left(D_{p}\right)$ ). Thus the valuation domain $D_{p}$ has principal maximal ideal and finite residue field $\mathrm{CC}$ Proposition I.3.16]. By Theorem 1.1, $\operatorname{Int}\left(D_{p}\right)$ is not a $\mathrm{P} v \mathrm{MD}$ and, from Proposition $2.3, \operatorname{Int}\left(D_{p}\right)=\operatorname{Int}(D)_{p}$. Hence $\operatorname{Int}\left(D_{p}\right)$ should be a $\mathrm{P} v \mathrm{MD}$ by [K1, Theorem 3.7] and we have reached a contradiction.

On the contrary, let us suppose that

$$
\operatorname{dim}\left(D_{p}\right)=1, \quad \text { for each } p \in \Theta_{0} .
$$

To prove that $\operatorname{Int}(D)$ is a $\mathrm{P} v \mathrm{MD}$ it is sufficient to show that $\operatorname{Int}(D)$ localized at each $t$-prime ideal $\mathfrak{P}$ is a valuation domain.

Corollary 2.5 provides a complete description of the $t$-spectrum of $\operatorname{Int}(D)$. The localizations of $\operatorname{Int}(D)$ at its $t$-primes are the following:

- if $\mathfrak{P}=\beta_{q}$, then $\operatorname{Int}(D)_{\beta_{q}}=K[X]_{q}$ and it is a valuation domain;

- if $\mathfrak{P}=J_{p}$, for $p \in \Theta_{1}$, then $\operatorname{Int}(D)_{J_{p}}=D_{p}[X]_{\left(p D_{p}[X]\right)}$ and it is a valuation domain since $p D_{p}[X]$ is a $t$-prime ideal of the $\mathrm{P} v \mathrm{MD} D_{p}[X]$;

- if $\mathfrak{P}=p_{\alpha}$, then $\operatorname{Int}(D)_{p_{\alpha}}=\left(\operatorname{Int}(D)_{p}\right)_{\left(p_{\alpha} \operatorname{Int}(D)_{p}\right)}=\operatorname{Int}\left(D_{p}\right)_{\left(p_{\alpha} \operatorname{Int}\left(D_{p}\right)\right)}$ and it is a valuation domain since $\operatorname{Int}\left(D_{p}\right)$ is a Prüfer domain.

To compute the $t$-dimension of $\operatorname{Int}(D)$ we go through the following considerations. No prime ideals of the type $\beta_{q}$ can be included in ideals of the type $J_{p}$. In fact,

$$
\beta_{q} D_{p}[X]=q K[X] \cap D_{p}[X] \nsubseteq p D_{p}[X]
$$

since $D_{p}$ is a valuation domain [Gi1, Theorem 19.15].

The ideals of the type $p_{\alpha}$ are maximal and of heigth, at most, 2. They can only contain $t$-primes of the type $\beta_{q}$ with $q(\alpha)=0$ [CC, Proposition V.3.3]. Thus the 
only possible saturated chains of $t$-primes in $\operatorname{Int}(D)$ are the following:

(i) $(0) \subset \beta_{q} \subset p_{\alpha}$, where $p \in \Theta_{0}$ and $q(\alpha)=0$.

(ii) (0) $\subset J_{p_{1}} \subset \cdots \subset J_{p_{n}}$, where $(0) \subset p_{1} \subset \cdots \subset p_{n}$ is a saturated chain of $t$-primes of $D$. The length of such a chain can be, at most, $t$-dim $(D)$.

The conclusion follows from the fact that $D$ is a $\mathrm{P} v \mathrm{MD}$ which implies that $t-\operatorname{dim}(D)=\sup _{p \in \Theta}\left\{\operatorname{dim}\left(D_{p}\right)\right\}$.

We observe that the finiteness-condition on the $t$-maximal ideals of $D$ (that is, each nonzero element $x$ of $D$ belongs to finitely many $t$-maximal ideals and which holds in Krull-type domains) is not, in general, necessary. It is possible to find a $\mathrm{P} v \mathrm{MD}$ such that $\operatorname{Card}(D / p)=\infty$, for all $p \in \Theta$ and which does not satisfy the above mentioned finiteness-conditon. Such a domain $D$ can be obtained by considering an almost Dedekind domain, which is not Dedekind, with all residue fields infinite; we refer, for instance, to Gi2 for this type of construction. In this case, $\operatorname{Int}(D)=\bigcap_{p \in \Theta} \operatorname{Int}\left(D_{p}\right)=\bigcap_{p \in \Theta} D_{p}[X]=D[X]$ is a $\mathrm{P} v$ MD [K1, Theorem $3.7]$.

This fact suggests that, for a general characterization, we do not have to ask that the intersection $\bigcap_{p \in \Theta} D_{p}$ is locally finite. More precisely, from Ch, Corollaire 1] we can write

$$
\begin{gathered}
\operatorname{Int}(D)=\bigcap_{p \in \Theta} \operatorname{Int}\left(D_{p}\right)=\left(\bigcap_{p \in \Theta_{0}} \operatorname{Int}\left(D_{p}\right)\right) \cap\left(\bigcap_{p \in \Theta_{1}} \operatorname{Int}\left(D_{p}\right)\right) \\
=\operatorname{Int}\left(\bigcap_{p \in \Theta_{0}} D_{p}\right) \cap\left(\bigcap_{p \in \Theta_{1}} D_{p}[X]\right)=\operatorname{Int}\left(D_{0}\right) \cap D_{1}[X],
\end{gathered}
$$

where $D_{0}:=\bigcap_{p \in \Theta_{0}} D_{p}$ and $D_{1}:=\bigcap_{p \in \Theta_{1}} D_{p}$. We call $D_{1}[X]$ the polynomial part of $\operatorname{Int}(D)$, and we say that it is not trivial if $D_{1} \neq K$. The existence of a nontrivial polynomial part, in the sense described above, represents a remarkable difference between the Prüfer and the P $v$ MD characterization of $\operatorname{Int}(D)$. In fact, if $\operatorname{Int}(D)$ is a Prüfer domain, then $D_{1}[X]$ is also Prüfer (being an overring of $\operatorname{Int}(D)$ ). But $D_{1}[X]$ is a Prüfer domain if and only if $D_{1}$ is a field. Thus if we suppose $\operatorname{Int}(D)$ to be Prüfer, then $D_{1}=K$. On the other hand, if $\operatorname{Int}(D)$ is a $\mathrm{P} v \mathrm{MD}$, then also $D_{1}[X]$ is $\mathrm{P} v \mathrm{MD}$ (because $D$ is a $\mathrm{P} v \mathrm{MD}$ and $D_{1}$ is an intersection of localizations of $D$ at $t$-prime ideals, so that $D_{1}$ is also a $\left.\mathrm{P} v \mathrm{MD}\right)$. But this is not a contradiction since the $\mathrm{P} v \mathrm{MD}$ property transfers from any domain $D$ to the polynomial ring $D[X]$, even if $D$ is not a field.

Therefore for the P $v$ MD's characterization we cannot exclude, a priori, the possibility that $\operatorname{Int}(D)$ has a nontrivial polynomial part, that is, $D_{1} \neq K$.

We now consider the overring of $D, D_{0}=\bigcap_{p \in \Theta_{0}} D_{p}$. We assume, as a necessary condition, that $D$ is a $\mathrm{P} v \mathrm{MD}$ (Proposition 3.1). If $D$ is a Krull-type domain, by Theorem 3.2, $\operatorname{dim}\left(D_{p}\right)=1$ and $D_{p}$ has principal maximal ideal and finite residue field (since $\operatorname{Int}\left(D_{p}\right) \neq D_{p}[X]\left[\mathrm{CC}\right.$, Proposition I.3.16]). Hence $D_{0}$ is a locally finite intersection of DVR's with finite residue fields, that is, $D_{0}$ is a Dedekind domain with finite residue fields. Therefore $\operatorname{Int}\left(D_{0}\right)$ is a Prüfer domain.

From this observation, in order to generalize this characterization to a generic domain $D$, we ask the following questions:

1) Should $\operatorname{dim}\left(D_{p}\right)=1$, for each $p \in \Theta_{0}$ ?

2) Should $\operatorname{Int}\left(D_{0}\right)$ be a Prüfer domain? 
The next result characterizes the Noetherian domains $D$ for which $\operatorname{Int}(D)$ is a $\mathrm{P} v \mathrm{MD}$, giving an analogue, for P $v \mathrm{MD}$ 's, of J.L. Chabert's characterization of Noetherian domains $D$ such that $\operatorname{Int}(D)$ is Prüfer.

Corollary 3.3. If $D$ is a Noetherian domain, then $\operatorname{Int}(D)$ is a PvMD if and only if $D$ is a Krull domain.

Proof. From Theorem 3.2, if $D$ is a Krull domain then $\operatorname{Int}(D)$ is a $\mathrm{P} v$ MD. If, on the contrary, $\operatorname{Int}(D)$ is a $\mathrm{P} v \mathrm{MD}$, then $D$ is a $\mathrm{P} v \mathrm{MD}$ by Proposition 3.1 , so that $D$ is integrally closed. Thus $D$ is a Noetherian, integrally closed domain, that is, $D$ is a Krull domain Gi1, Theorem 43.4].

A one-dimensional Krull domain is Dedekind and we easily have the following corollary:

Corollary 3.4. Let $D$ be a one-dimensional Noetherian domain. Then, $\operatorname{Int}(D)$ is a PvMD if and only if $D$ is a Dedekind domain.

\section{REFERENCES}

[CC] P.J. Cahen - J.-L. Chabert, Integer-Valued Polynomials, Math. Surveys and Monographs (AMS) 48 (1997). MR 98a:13002

[CH] P.-J. Cahen - Y. Haouat, Polynômes à valeurs entières sur un anneau de pseudo-valuation, Manuscripta Math. 61 (1988), 23-31. MR 89f:13007

[Ch] J.-L. Chabert, Anneaux de polynômes à valeurs entières et anneaux de Fatou, Bull. Sc. Math. France 99 (1971), 273-283. MR 46:1780

[Gi1] R. Gilmer, Multiplicative Ideal Theory, Marcel-Dekker, New York (1972). MR 55:323

[Gi2] R. Gilmer, Prüfer domains and rings of integer-valued polynomials, J. Algebra 129, n.2 (1990), 502-517. MR 91b:13023

[Gr] M. Griffin, Some Results on v-multiplication Rings, Canad. J. Math. 10 (1967), 710-722. MR 35:6665

[HZ] E.G. Houston - M. Zafrullah, Integral Domains in which each t-ideal is divisorial, Michigan Math. J. 35 (1988), 291-300. MR 89i:13027]

[J] P. Jaffard, Les Systèmes d'Idéaux, Dunod, Paris (1960). MR 22:5628

[K1] B.G. Kang, Prüfer $v$-Multiplication Domains and the Ring $R[X]_{N_{v}}$, J. of Algebra 123 (1989), 151-170. MR 90e:13017

[K2] B.G. Kang, Some questions about Prüfer v-Multiplication Domains, Comm. Algebra 17(3) (1989), 553-564. MR 90a:13033

[L] A. Loper, A classification of all D such that Int $(D)$ is a Prüfer domain, Proc. Amer. Math. Soc. (to appear).

Faculté des Sciences de Saint-JÉrôme, Université D’Aix-Marseille III, 13397 MarSeIlle, France

E-mail address: francesca.tartarone@vmesa12.u-3mrs.fr

Current address: Dipartimento di Matematica, Università degli Studi di Roma "Roma Tre", Largo Murialdo 100146 Roma, Italy

E-mail address: tfrance@matrm3.mat.uniroma3.it 